\title{
Die Medizin im Wallis bis zum Ausgang des 19. Jahrhunderts
}

Von Alfred Gottfried Kauertz

Wie ein Blatt liegt das Wallis in die Alpen eingebettet. Die Hauptrippe bildet das Rhonetal, mit der Blattspitze am Gotthardstock und dem Stielende am Genfersee. Das Tal mit seinen Viertausendern schiebt sich wie ein Riegel zwischen die Nord-Süd-Verbindung und blockiert scheinbar den Transitverkehr. Daß dem nicht so ist, beweist die frühe Besiedlung des Tales und seiner Seitentäler schon in der jüngeren Steinzeit. In der Eisenzeit lassen sich die ersten Stammesgruppen nachweisen. Im Westen nach Gallien hin waren es die Nantuaten, im Gebiet des Großen St.Bernhard siedelten sich die Veragrer an. Um die heutige Hauptstadt Sitten haben sich die Seduner niedergelassen und im Oberwallis schließlich die Uberer. Die Namengebung Rhodanus scheint ligurischen Ursprungs zu sein, und heute noch wird von den Deutschwallisern die Rhone Rotten genannt. Nach ersten Mißerfolgen um 57 vor Christus unter Cäsar erfolgte die römische Eroberung des Wallis um 15 und 10 vor Christus. Die Bezeichnung 'Penninische Alpen' stammt von einer lokalen Gottheit namens Penn.

Ein medizinhistorisch wichtiger Fund wurde in einem neolithischen Grab bei La Barmaz bei Collombey im Unterwallis gemacht. Es handelt sich um einen trepanierten Schädel, dessen Kallusränder auf eine nur kurze Überlebensdauer des Operierten hinweisen. Aus römischer Zeit ist eine Taschenapotheke aus Elfenbein gefunden worden. Der Schiebedeckel zeigt Äskulap mit seiner Tochter Hygieia; der Kasten weist elf unterschiedlich große Fächer auf. Aus dieser Epoche stammen noch Salbentöpfchen, Becher aus Bergkristall zur Medikation und ein römisches Chirurgenbesteck. Diese Funde sind im Museum Valeria in Sitten zu sehen.

Für mehr als ein Jahrtausend versiegen die Quellen, die das Bild der Medizin im Wallis speisen könnten. Erst mit der Klerikermedizin tauchen weitere Nachrichten auf. Ein Carterius Johannes aus Evian (Savoyen), der 1366 Domherr in Sitten war, wird um 1360 als 'magister in artibus et in medicina' erwähnt. Ebenfalls ein Kleriker war der 'physicus' Wilhelm Perronet aus Leuk, der 1343 als Zeuge erwähnt wird.

Zur Römerzeit stand bereits auf dem Großen St.Bernhard ein 'hospitium', während das heutige Hospiz im 11.Jahrhundert vom heiligen Bernhard von Menthon gegründet wurde. Das zweite Hospiz wurde von den Rittern des Johanniterordens um 1235 auf dem Simplon errichtet und in diesem Jahre zum ersten Male erwähnt. Auch in den städtischen Ansiedlungen des Tales entstanden nach 
und nach Hospize, die für Wanderer und Pilger mit kurzem Aufenthalt gedacht waren. Das Bürgerspital von Sitten ist eine Gründung aus dem 12. Jahrhundert. Als feste Einrichtungen waren die Thermalquellen von Leukerbad und Brigerbad bereits zur Römerzeit bekannt, und sie hatten auch im Mittelalter regen Zulauf. Der Walliser Felix Platter, Professor in Basel, berichtet über einen Besuch in Leukerbad recht anschaulich.

Die Ausbildung der Walliser Ärzte erfolgte im Ausland. Für die Unterwalliser waren Montpellier und später Paris die großen Schulen, die sie aufsuchten. Die Oberwalliser zog es mehr nach Wien, München, Freiburg im Breisgau und nach Würzburg. Nachforschungen über Walliser Studenten an ausländischen Universitäten sind etwas schwierig, da man sich hauptsächlich um die Kleriker unter ihnen bemühte, denn das Wallis ist ja ein rein katholischer Kanton. In Heidelberg ist ein gewisser Petrus Burgener aus Visp zu finden, der sich am 12. November 1788 als Medizinstudent dort eingetragen hat (diese Eintragungen umfassen den Zeitraum von 1386 bis 1807). Doch hat es dieser Oberwalliser nicht weit gebracht. Eine Notiz besagt, daß Burgener im Herbst 1790 flüchtig wurde, weil er durch seine tollen Streiche den Universitätsbehörden 'ordentlich viel zu schaffen gegeben habe'. Aus Wien werden 1721 und 1783 Medizinstudenten mit Walliser Namen genannt.

Als Folge der ungenügenden medizinischen Versorgung des Wallis spielte die Volksheilkunde eine große Rolle. Die akademisch gebildeten Ärzte hatten ihre Praxen in den Städten des Rhonetales. Daher war es nötig, daß die Kranken zu ihnen kommen oder transportiert werden mußten. Andererseits machten die Ärzte stunden- und tagelange Fuß- oder Maultierreisen in die abgelegenen Winkel der Seitentäler. Im Jahre 1812 gab es im Oberwallis nur zwei Chirurgen mit Sitz in Visp, die auch zuständig für das Saas- und Mattertal waren. Das Goms bekam erst im Jahre 1869 seinen ersten Arzt. Eine Aufstellung der praktizierenden Ärzte im Wallis zeigt, daß während hundert Jahren die ärztliche Versorgung praktisch gleich blieb. $1812 \mathrm{kam}$ auf 3737 Einwohner je einer der siebzehn Ärzte. Deren Zahl erhöhte sich bis 1910 auf siebenunddreißig; aber die Bevölkerung hatte zugenommen, so daß ein Arzt 3729 Einwohner zu betreuen hatte.

Bei diesen Verhältnissen blühte die Kurpfuscherei, mit der sich schon sehr früh der Landrat beschäftigen mußte. Die Obrigkeit verlangte von allen in der Medizin tätigen Personen Zeugnisse, Lehr- oder Meisterbriefe, mit denen sie sich als mit der Medizin vertraut ausweisen konnten. Anderenfalls drohte Leibesstrafe sowie Verlust an Hab und Gut. So lautete der Landratsbeschluß vom 16. Dezember 1615, der am 25. Mai 1633 verschärft nachgedoppelt wurde.

Andererseits gab es unter den Laienärzten geschickte Leute, die sehr beliebt 
waren. Carl Müller berichtet davon in seinem Beitrag 'Volksmedizinisch-geburtshilfliche Aufzeichnungen aus dem Lötschental' anschaulich. Dort nahm ein Wundarzt einen Kaiserschnitt vor, der bis auf die Nahtversorgung ausgezeichnet verlief. Die Laienärzte erlernten ihr Handwerk an den Beinbrüchen der Haustiere. Da hauptsächlich in den Bergregionen seit altersher Viehzucht betrieben wurde, dürfte es eine Vielzahl von empirisch geschulten Wundärzten gegeben haben. Der gebrochene Knochen wurde durch Zug und Gegenzug in die richtige Lage gebracht. Geschmolzenes Harz von Tannen und Arven wurde auf Tuchlappen gegossen und diese um die zu fixierende Stelle gebunden. Beim Erkalten erstarrte die Harzmasse und ergab so einen festen Verband, der zusätzlich durch Holzschienen gefestigt wurde. Genau so ging man beim Menschen vor, ein billiges, einfaches und doch sicher erfolgreiches Verfahren, das schon vor Jahrhunderten angewendet wurde.

Manche dieser mit 'Doktor' angeredeten Personen waren recht gescheit und konstruierten Apparaturen, die ebenso genial wie einfach waren. Beispielsweise eine Schiene für Unterschenkelfrakturen, eine Art Schaukel, im Wallis 'Tröglein' genannt, deren Aushöhlung genau der Wade und den Knöcheln angepaßt war. Die Seitenwände konnten verschoben und festgeschraubt werden. Diese Schaukel wurde mit vier Schnüren über dem Fußende des Bettes aufgehängt. Bewegte sich der Patient, so schmerzte das nicht sehr, da der gebrochene Unterschenkel ja fixiert war und die Aufhängevorrichtung den Bewegungen des Kranken folgte.

Oft vererbte sich das ärztliche Handwerk vom Vater auf den Sohn. Auf diese Weise gab es in manchen Dörfern regelrechte 'Doktorsfamilien'. So stammt das vorhin beschriebene 'Tröglein' von einem alten 'Doktor', der es von seinem Vater und Großvater übernommen hatte.

Der Regierung in Sitten waren diese Bräuche bekannt, und sie unterstützte sogar dieses Handwerk, weil gebildete Ärzte kaum in Bergdörfern hausen wollten. Ein letztes Gesetz zu dieser Frage kam am 24. November 1849 heraus. Es erlaubte den 'Dokters' die Behandlung und Heilung von Brüchen und Verrenkungen. Allerdings durften sie keine Medikamente abgeben; die Innere Medizin sollte den approbierten Ärzten vorbehalten bleiben.

Neben diesen rühmlichen Ausnahmen hat es doch viele Pfuscher gegeben. Mit ihnen mußten sich Ärzte und Behörden herumplagen. 1850 sprach man einen angeklagten Kurpfuscher frei, und prompt demissionierte der Arzt Tenisch von Mörel aus Protest als Bezirksarzt. Und im Jahre 1863 war es sogar der gesamte Walliser Sanitätsrat, der zurücktrat, weil man einen bereits vorbestraften italienischen Wunderheiler nicht ausweisen wollte. Nun, die wachsende Zahl der Ärzte hat dieses Problem zwar entschärft, aber nicht beseitigt. Denn noch heute prak- 
tizieren im Wallis Kräuterweiblein und Naturheiler, und die Leute suchen sie gerne auf, wenn angeblich die Schulmedizin keinen Rat mehr weiß.

Zahnärzte gab es im Wallis immer wenig. Erstmals wird im Jahre 1615 ein 'Zahnbrecher' erwähnt. 1864 ließ sich der Genfer Cané im Wallis nieder, und 1889 durfte auch Henri Bujard auf Grund eines waadtländischen Diploms seine Kunst im Wallis ausüben. 1897 erhielt der erste Walliser - Joseph Debon von Sitten sein zahnärztliches Diplom. So waren die Bewohner des Wallis gezwungen, zur Selbsthilfe zu schreiten. Irgendein Beherzter fand sich immer, Zähne zu brechen. Vielfach benutzte er dazu selbstgeschmiedete Zahnwerkzeuge, wie Adolf Roos in seiner Studie 'Die Zahnkaries der Gomserkinder' berichtet. Andererseits hatten gerade die Gomser ausgezeichnete Zähne und Gebisse, die selten Eingriffe nötig machten. Die einfache, kräftige Kost, besonders das selbstgebackene Roggenbrot, und das fast völlige Fehlen künstlicher Schleckereien kam ihren Zähnen zugute.

Über die Veterinärmedizin ist hierzulande wenig bekannt, obwohl die Viehwirtschaft eine sehr große Rolle spielte. Die ersten beiden Tierärzte werden 1863 aufgeführt.

Ähnlich verhält es sich mit den Apotheken. Sicher gab es bis zum 19. Jahrhundert nur im Unterwallis Apotheken, denn die erste im Oberwallis wird 1865 erwähnt, und um 1900 waren es hier erst drei. In Visp, Brig, Leuk und Siders gab es lediglich Arzneimitteldepots für die Oberwalliser Ärzte.

Auch über die Hebammen läßt sich vor 1800 nichts Sicheres sagen. Es gab sie, denn die Pfarrherren hatten die Pflicht, dem Bischof zu melden, ob die Dorfhebamme zuverlässig sei und taufen könne. Meist wurden die Nachbarinnen um den Dienst an der Gebärenden gebeten. Den diplomierten Hebammen begegnete man seltsamerweise mit Mißtrauen. Es kam schließlich so weit, daß der Staat die Laienhebammen und die Familien bestrafen mußte, die undiplomierte Hebammen beigezogen hatten. Noch bis 1919 gab es im Wallis Hebammenschulen, die am Wohnsitz des Arztes eingerichtet waren, der als Kursleiter fungierte. Geradezu legendären Ruf hat eine dieser in einem Kurs in Visp ausgebildeten Hebammen im Lötschental. Es ist Marjosi Tannast, die im Jahre 1883 erstmals selbständig einer Gebärenden beistand.

Die großen Seuchen machten auch vor dem Wallis nicht halt. Die Pest hat hier ziemlich stark gewütet. 1349 starben in Sitten drei Viertel der Einwohner; 1463 erfolgte ein erneuter Ausbruch, ebenso im 16. und 17.Jahrhundert. Der Oberwalliser Geschichtsforscher Peter Arnold, alt Pfarrer zu Mörel, schildert die Pestausbrüche in Gondo-Zwischbergen an der italienischen Grenze. Dort tauchte die Pest erstmals 1502 auf. 1513 waren in Gondo 113 Pesttote zu beklagen, und 
genau ein Jahrhundert später, 1613, werden zwei Pestausbrüche gemeldet, die allein in Domodossola 313 Tote forderten. So wurde die Grenze gesperrt und die Quarantäne verhängt.

Im Staatsarchiv in Sitten liegen Berichte über Epidemien zwischen 1804 und 1867 sowie von 1831 bis 1836 vor. Da wird von Fièvre jaune und Choléra geschrieben. Da ich bei Gelbfieber keine exakte Krankheitsbeschreibung finde, denke ich eher an die Weilsche Krankheit als Tierstallinfektion, die mit Ikterus einhergeht. Im Wallis kommt die Gelbfiebermücke nicht vor, und die Aedes aegypti bleibt ja nur bei ständig hohen Temperaturen infektiös. Es ist auch an Feldfieber zu denken, das angesichts der damals noch nicht korrigierten Rhone durchaus grassiert haben könnte. Und schließlich könnte es sich bei den choleraähnlichen Infektionen um die Cholera nostra, den Paratyphus, gehandelt haben, der heute noch im Wallis häufiger vorkommt. Doch diese Theorie bedarf einer gründlicheren Abklärung.

\section{Literatur}

Arnold, Peter, Licht und Schatten in den zehn Gemeinden von Östlich-Raron, 1961.

Arnold, Peter, Gondo-Zwischbergen an der Landesgrenze am Simplonpaß, 1968.

Bazala, Vladimir, Zur Geschichte des Kaiserschnittes. In: Die Waage, Bd. 4/2 (1965), S. 61.

Bloetzer, H., Lötschen und sein Prior, 1965.

Heierli, Jakob/Oechsli, Wilhelm, Urgeschichte des Wallis, 1896.

Grand, A., Walliser Studenten auf ausländischen Universitäten, 1910.

Maisen/Gattlen, Verzeichnis der an der Universität Wien immatrikulierten Walliser Studenten (1377-1794), 1967.

Imesch, D., Die Werke der Wohltätigkeit im Kanton Wallis, 1901.

Häfliger, Josef Anton, Pharmazeutische Altertumskunde und die Schweizerische Sammlung für historisches Apothekenwesen an der Universität Basel, 1937.

Roos, Adolf, Die Zahnkaries der Gomserkinder, 1937.

Studer, German, Das Medizinalwesen im Wallis von 1798 bis 1930, 1940.

Staatsarchiv Sitten Nr. 173/174.

Touring-Club der Schweiz, Unbekannte Schweiz: Wallis, o. J.

\section{Summary}

The author gives a survey of the medicine in the Swiss Canton Wallis from neolithic age until the 19 th century.

Alfred G. Kauertz

Kapuzinerstraße 10

CH-3902 Glis 